\title{
THE EFFECTS OF FEEDBACK-BASED INTERVENTION IN ERRORS DUE TO INAPPROPRIATE STORAGE AND TRANSPORTATION OF BLOOD BAGS IN HOSPITAL
}

Shokoufeh Aalaei ${ }^{1}$, Shahram Amini ${ }^{2}$, Zhila Taherzadeh ${ }^{3}$, Sanaz Khoshrounezhad ${ }^{1}$, Farnaz Khoshrounezhad ${ }^{1}$, Mohammad Reza Keramati ${ }^{4}$, Hadi Shahraki ${ }^{5}$, Saeed Eslami ${ }^{7,3,6^{*}}$

1: Student Research Committee, Department of Medical Informatics, Faculty of Medicine, Mashhad University of Medical Sciences, Mashhad, Iran

2: Department of Anaesthesiology, School of Medicine, Mashhad University of Medical Sciences, Mashhad, Iran

3: Targeted Drug Delivery Research Center, School of Pharmacy, Mashhad University of Medical Sciences, Mashhad, Iran

4: Cancer molecular pathology research center, School of Medicine, Mashhad University of Medical Sciences, Mashhad, Iran

5: Department of Electrical Engineering, Faculty of Engineering, University of Birjand, Birjand, Iran

6: Department of Medical Informatics, University of Amsterdam, Amsterdam, The Netherlands

7: Department of Medical Informatics, Faculty of Medicine, Mashhad University of Medical Sciences, Mashhad, Iran

\section{Correspondence:}

Saeed Eslami, Tel: +98-51-38002 2429; email: EslamiS@mums.ac.ir

\section{TYPE OF ARTICLE: CONFERENCE ABSTRACT}

\begin{abstract}
Introduction: Storage and transportation of red blood cells (RBCs) outside the standard range temperature as defined by guidelines can lead to hemolysis. One of the main factors believed to cause hemolysis is temperature. Infusion of corrupted RBCs leads to haemolytic reactions, which are severe and life threatening. The clinical implications of limited supply of red blood cells (RBCs) for transfusion can lead to the application of various strategies to decrease blood wastage and safely and effectively deliver the blood products. This study aims to evaluate the effects of feedback-based intervention on inappropriate temperature during storage and transportation of blood bags.

Methods: A quasi-experimental pretest-posttest design with a nonequivalent control group was performed on 200 RBC bags transported from the blood bank to the cardiac surgery intensive care unit (CSICU) and heart operating room (OR) for transfusion. To monitor the temperature of RBCs, temperature monitoring systems were attached to individual bags of RBCs. The temperature monitoring system was equipped with an accurate sensor and a memory to save temperature in the specified time interval (one per 2 minutes). Also, a form was designed to collect the required information. Each part of the form was completed by trained personnel in the six different phases of blood bag delivery and storage. The intervention group comprised of personnel in the blood bank department who received the intervention, including feedback about inappropriate temperature in the blood bank during the pre-intervention period. The control group included personnel and nurses in the OR, CSICU, or transportation who received no feedback. The comparison between the percentage with an inappropriate temperature before and after the implementation of the intervention was performed with a Pearson's chi-square test.

Results: The temperature monitoring devices recorded a total of 119,511 and 160,562 measurements (one per 2 minutes) in pre- and post-intervention periods, respectively. The percentage of inappropriate temperature for preand post-intervention period was $9 \%$ and $7.5 \%$ of total measurements, respectively. The percentage of errors in the blood bank decreased from $30 \%$ during the pre-intervention period to $12 \%$ during the post-intervention period $(P<0.05)$, representing a relative reduction of $60 \%$. Notably, there was an increase in the percentage of errors compared with the pre-intervention period observed in the phases related to the control group, which included the
\end{abstract}

\section{Abstracts of First National Congress of Medical Informatics, Mashhad, Iran, February 2017}

(C) 2017 The Authors. This is an open access article under the terms of the Creative Commons Attribution-NonCommercialNoDerivs License, which permits use and distribution in any medium, provided the original work is properly cited, the use is non-commercial and no modifications or adaptations are made. 
OR (from $6.5 \%$ to $20.5 \% ; p<0.05$ ), CSICU (from $1 \%$ to $2.5 \% ; p>0.05$ ) and transportation (from $0.6 \%$ to $16.6 \%$; $p<0.05)$.

Conclusion: The implementation of feedback-based intervention to increase awareness of the staff of critical conditions can have a dramatic impact in reducing inappropriate temperature of blood bags during storage and transportation.

KEYWORDS: Blood transfusion, Red blood cell, Blood quality, Inappropriate temperature, Feedback 